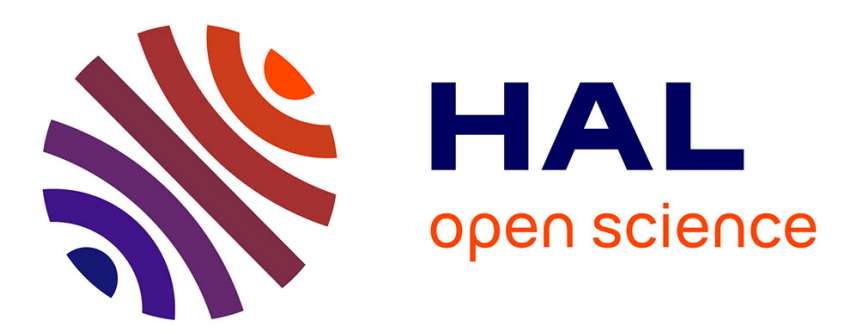

\title{
Rayleigh-Taylor-like instability in a foam film
}

Evgenia Shabalina, Antoine Bérut, Mathilde Cavelier, Arnaud Saint-Jalmes, Isabelle Cantat

\section{To cite this version:}

Evgenia Shabalina, Antoine Bérut, Mathilde Cavelier, Arnaud Saint-Jalmes, Isabelle Cantat. Rayleigh-Taylor-like instability in a foam film. Physical Review Fluids, 2019, 4 (12), 10.1103/PhysRevFluids.4.124001. hal-02394794

\section{HAL Id: hal-02394794 https://hal.science/hal-02394794}

Submitted on 12 Feb 2020

HAL is a multi-disciplinary open access archive for the deposit and dissemination of scientific research documents, whether they are published or not. The documents may come from teaching and research institutions in France or abroad, or from public or private research centers.
L'archive ouverte pluridisciplinaire HAL, est destinée au dépôt et à la diffusion de documents scientifiques de niveau recherche, publiés ou non, émanant des établissements d'enseignement et de recherche français ou étrangers, des laboratoires publics ou privés. 


\title{
Rayleigh Taylor like instability in a foam film
}

\author{
Evgenia Shabalina, Antoine Bérut, Mathilde Cavelier, Arnaud Saint-Jalmes and Isabelle Cantat \\ Univ Rennes, CNRS, IPR (Institut de Physique de Rennes) - UMR 6251, F- 35000 Rennes
}

(Dated: December 13, 2019)

\begin{abstract}
It is well known, since the seminal work of Mysels et al., that the thinner parts of a foam film go up by gravity, whereas the thicker parts go down. Preparing a foam film in a controlled way, so that the top part of the film is much thicker than the bottom part at initial time, we show that this situation is indeed unstable under gravity. The observed instability is identified as a Rayleigh-Taylor like instability and studied in the linear regime. The wavelength and the growth rate are measured as a function of the effective gravity, and as a function of the thick film extension. We theoretically show that all wavelengths are unstable, as the surface tension stabilizing the small wavelengths in the classical bulk Rayleigh Taylor instability has no significant equivalent in a foam film. The fastest mode is analytically determined and is in qualitative agreement with the experimental observations.
\end{abstract}

\section{INTRODUCTION}

Inside an aqueous foam, gas bubbles are packed and separated by thin liquid films. The existence of such a foam directly relies on the ability to stabilize these films at a finite thickness. For that purpose, surfactants need to be added to the aqueous phase: they can then adsorb on the gas-liquid interfaces, and eventually provide some stabilizing mechanisms.

In parallel, both when a foam ages and when it flows, the arrangement of the bubbles does not remain still, and bubbles keep swapping their relative positions. This implies that the liquid films are contracted, elongated, deformed and that some films vanish while others are created. As a consequence, some liquid flows are induced inside the films, and the liquid is dynamically distributed between the films and the menisci to which they are connected (also known as 'Plateau borders').

The liquid flow in such surfactant-stabilized films has some specific features. On one hand, the liquid is strongly confined between the two interfaces covered by surfactants, and any flow relatively to these interfaces is highly dissipative; this effect appears to be a key element for understanding the high effective viscosity of liquid foams $[1,2]$. On the other hand, the in-plane mobility of the liquid is very high, so that turbulent flows can be easily induced in dedicated experimental configurations. In consequence, surfactant-stabilized liquid films have been used as a model system to reach high Reynolds numbers and to study turbulence in 2D [3, 4].

For all these reasons, understanding all the equilibrium and dynamical properties of an aqueous foams requires to unravel the interactions and flows acting at the scale of the liquid films. Many experimental results have been collected by monitoring a single liquid film. A large literature is available on horizontal films held on solid frames, especially in the framework of the 'thin film balance' apparatus $[5,6]$. With this setup, one can focus on the small thickness regime (due to high capillary suction by the meniscus), and on the repulsive and attractive forces between the two surfactant-covered layers. Over the years, such experiments have brought many new insights on the required conditions for film stability, as well as on confinement effects (like stratification, $[5,6]$ ). However, gravity plays no role in these horizontal films, and only smooth radial flows - due to capillary suction towards the surrounding meniscus - can be monitored. Thus, this setup is not suited for investigating flows induced during fast dynamical changes of film area, like those occurring during bubble swapping.

On the opposite, other experiments have been performed on single vertical films, most generally held on large (centimeters) solid frames. In such conditions, the gravitational drainage of a film can be studied [7-11]. Subtle effects, like 'pinching' or 'marginal regeneration' $[7,12]$, have then been evidenced, illustrating the nontrivial flows occurring in such vertical films. It is indeed well known since the seminal work of Mysels [7] that thinner parts of a foam film move up by gravity, whereas the thicker parts go down. A continuous injection of surfactant solution inside a vertical film can also be done to sustain high film thickness of a few microns. As pointed out previously, 2D turbulence has actually been studied with such sustained vertical films [3, 4]. Nevertheless, these films are then far from those found in usual foams, both in terms of size, thickness and velocity scales.

More recently, to fill the gap between a single isolated film and a 3D opaque foam, experiments have been performed on system which can be considered as the elementary building blocks of a foam, meaning a few films connected to one (or a few) meniscus [13-20]. The main goal is then to identify non-trivial features occurring at the connections between free films and menisci. Together with the fact that these clusters of films are relatively easy to build, one of their advantages is that they allow to visualize and to monitor the various flows, deformations and out-of-equilibrium film thicknesses [21]. Another advantage is that these elementary clusters can be activated on purpose: one can trigger re-organization of films, control flows in films, etc... Also - as shown in the following article - one can also easily change the setup orientation to tune the effects of gravity. In parallel, by continuing to study such clusters of films and menisci, one can also expect to shed light on the efficiency of these elementary building blocks to mimic 3D foams. In fact, how far one can transpose the observations made on film clusters to 
any foams remains to be fully elucidated, especially in terms of bubble sizes, liquid fractions, or chemical formulation.

Here, we present results performed on a new type of film/meniscus cluster, and allowing us to prepare one of the foam film in a controlled way, so that the top part of this film is initially much thicker than the bottom part. We show that this situation is indeed unstable under gravity and we identify this instability as a Rayleigh Taylor instability [22]. The wavelength and the growth rate are measured in the linear regime as a function of the effective gravity, and as a function of the film extension. We built a model involving viscous, gravitational, and inertial contributions. The fastest mode is analytically determined and is in qualitative agreement with the experimental observations.

\section{EXPERIMENTAL SET-UP}

The set-up and the reference frame are schematized in Fig. 1. A horizontal free meniscus of width $w=5 \mathrm{~cm}$ is connected to three flat rectangular foam films. For one of these films, denoted by $F$ in the following, the edge parallel to the free meniscus can translate along the lateral edges, and its position is controlled by a piezo translation stage. Its area is thus $w \times L(t)$ with $L(t)$ the distance between the moving edge and the free meniscus. The two other films $F^{\prime}$ and $F^{\prime \prime}$ are of area $w \times L_{l a t}$, with $L_{l a t}=13 \mathrm{~mm}$.

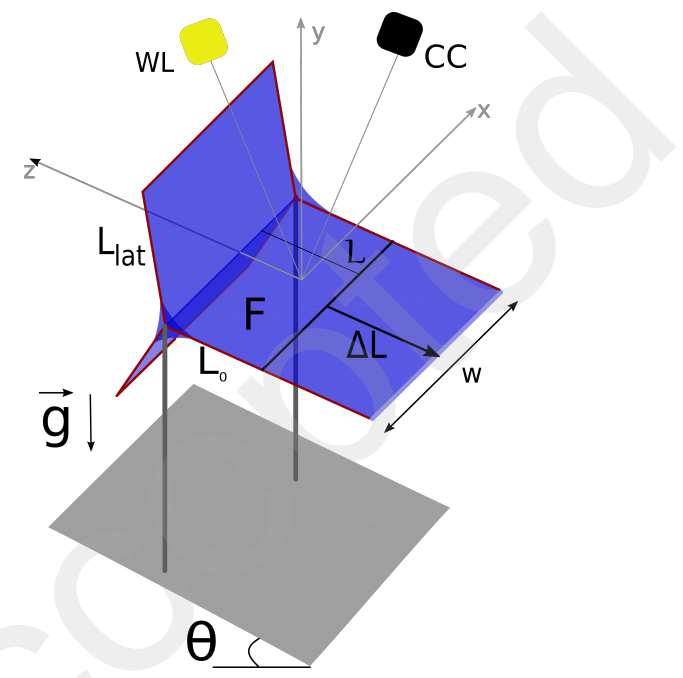

FIG. 1. Scheme of the experimental set-up and notations used in the text. Light blue represent the three foam films connected along the free meniscus (dark blue). They are supported by a deformable metallic frame : the red lines are the immobile edges and the black line is the mobile edge. The deformable film $F$ is illuminated with the white light WL and recorded in reflection with the color camera CC. The whole set-up is on a table which can be tilted by an angle $\theta$.

We define as $x$ the meniscus direction, and as $(x, z)$ the plane of the film of interest $F$. We use two variants of the same set-up. The first one (set-up A) is on a table which inclination $\theta$ can be controlled in the range $\left[0-9^{\circ}\right]$, so that the direction $z$ makes an angle $\theta$ with the horizontal. With these conventions, the projection of the gravity vector on the film plane is $-\bar{g} \mathbf{e}_{\mathbf{z}}$, with $\bar{g}=$ $g \sin \theta$. In the second one (set-up B), the film $F$ is placed vertically, with the free meniscus at the top, so $\theta=\pi / 2$. We describe below the protocol for set-up (A), the one for set-up (B) being similar.

We used two different foaming solutions. Solution $S_{a}$ is made of sodium dodecyl sulfate (SDS) at $5.6 \mathrm{~g} / \mathrm{L}$ and glycerol at $15 \%$ of volume. In solution $S_{b}$ we added dodecanol at $0.05 \mathrm{~g} / \mathrm{L}$. The bulk viscosity is $\eta=1.510^{-3}$ Pa.s, the density is $\rho=1.0510^{3} \mathrm{~kg} / \mathrm{m}^{3}$ and the surface tension is $\gamma=29 \mathrm{mN} / \mathrm{m}$ for the $S_{a}$ and $30 \mathrm{mN} / \mathrm{m}$ for $S_{b}$ [23]. The films are prepared by immersing the frame into a vessel containing the foaming solution and by removing slowly the vessel. The film drains during $15 \mathrm{~s}$, then is extended at $\mathrm{t}=0$, by moving the piezo stage at velocity $U_{m}=80 \mathrm{~mm} / \mathrm{s}$, so that $L(t)$ varies from $L_{0}=6 \mathrm{~mm}$ to $L_{0}+\Delta L=19 \mathrm{~mm}$. The film $F$ is observed at a frame rate of $60 \mathrm{~Hz}$ with a color camera Imaging Source DFK 23 UM021, with $1280 \times 960$ pixels. The incident light is in the $(\mathrm{x}, \mathrm{y})$ plane and the camera is at the mirror position.

A spectral camera Resonon Pika L has also been used to record the light spectrum reflected by the film $F$, along a line perpendicular to the free meniscus. As discussed in section II B, this provides the film thickness.

\section{A. Qualitative description of the flow}

An example of film evolution during and after the displacement of its moving edge is shown in Fig. 2, for the case of set-up (B). The images are all cropped just below free meniscus (see the few black lines at the top of the images). This meniscus remains almost at a constant position during the deformation. The moving edge appears in black at the bottom of the film. It begins to move at image (a) and stops at image (d).

The shape of the film $F$ remains flat and rectangular and its area $w L(t)$ increases at the rate imposed by the linear stage motion. As already shown in [17], this area variation is first insured by an extension of the foam film initially present (denoted as the initial film in the following), which lead to the increase of the surface tension. When the film tension becomes large enough, the initial film becomes able to pull on the meniscus and to extract volume and interface from this reservoir: new pieces of film, hereafter called the Frankel's films, are extracted from the menisci bounding the deformed film [7].

Consistently, the initial film is first stretched and get thinner between images (a) and (d). Colors indicate for example a thickness of $450 \mathrm{~nm}$ in (b) (dark blue), and $350 \mathrm{~nm}$ in (d) (yellow). In image (c) the gray bands appearing at the top and bottom are the Frankel's films extracted from the menisci. They are thus much thicker 


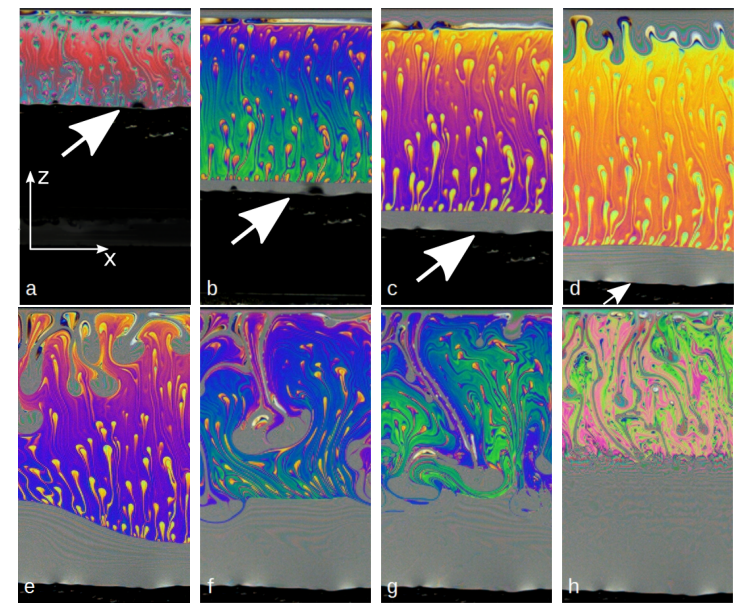

FIG. 2. Images of the film at the times [0,67, 83, 167, 250, $284,384,1467] \mathrm{ms}$, in the vertical case, solution $S_{b}$. In the $z$ direction, the whole height is shown, from the moving meniscus at the bottom (see the white arrow on images (a-d)) to the free meniscus at the top of the image. In the $x$ direction, the image is cropped in the central part of the film, and only $10.3 \mathrm{~mm}$ are visible. The remaining part of the film evolves similarly, excepted close to the lateral sides. The bottom edge moves over $13 \mathrm{~mm}$ during the first $100 \mathrm{~ms}$ (images $(\mathrm{a}-\mathrm{d}$ )), at a velocity of $80 \mathrm{~mm} / \mathrm{s}$.

than the initial film and a sharp frontier separates them from it.

The Frankel's film extracted from the bottom meniscus (located on the moving edge) remains stable, whereas the one extracted from the top meniscus (the free one) destabilizes after a fraction of second. Its frontier with the initial film exhibits a relatively regular and sinusoidal shape for a while (image (d)) and then produces drop-like structures reminiscent of a usual Rayleigh Taylor instability (images (e) and (f)). These drops of thick film eventually detach from the top Frankel's film and fall through the initial film until they merge with the bottom Frankel's film (images (g) and (h)).

In this paper, we focus on the linear regime of the gravitational destabilization of the top Frankel's film. Note that, even before the motor motion, some spots move up in the film by a process known as marginal regeneration [7]. They are thinner than the surrounding film, and produced at the frontier between the menisci and the thin film, by marginal pinching [12]. As the thickness difference is small, their upward velocity is smaller than the velocity of the instability, as observed qualitatively by following patterns in Fig. 2. They do not influence the dynamics of interest.

\section{B. Image processing and geometrical characterizations}

The thicknesses of both the initial film and the Frankel's film are deduced from the data obtained with a spectral camera. This camera makes the image of a line $\mathcal{L}$ perpendicular to the free meniscus and measures the spectrum of the light reflected by each point of $\mathcal{L}$ : the intensity $I$ of the light of wavelength $\lambda_{j}$, in the range $[375-1010]$, reflected by the piece of film at the position $z_{i}$ and of size $d z d x$ is recorded by the pixel $(i, j)$ of the $2 \mathrm{D}$ sensor. The distance $d x$ is fixed by the width of a slit in the camera and $d z=54 \mu \mathrm{m}$ is the spatial resolution. The light trajectory is in the plane $(y, z)$ and the incidence angle on the film is $\phi=45^{\circ}$. The lamp is a usual halogen lamp. A typical image obtained after motor motion for the case $\theta=9^{\circ}$ is shown in Fig. 3a.

We measure the thickness profile $h(z)$ on the last image before the destabilization becomes visible using the relation

$$
I(\lambda) \propto 1-\cos \left(\frac{4 \pi h n}{\lambda}\left(1-\frac{\sin ^{2} \phi}{n^{2}}\right)^{1 / 2}\right),
$$

with $n$ the optical index of the solution.

As shown in Fig. 3b, the thin and thick parts of the film are separated by a sharp transition occurring over a distance $\delta \sim 100 \mu \mathrm{m}$ where the thickness is not resolved because the gradient is too large. The average thicknesses in the thin and thick parts are denoted respectively by $h_{1}$ and $h_{2}$.

The experiments with the vertical set-up have been performed without the spectral camera and the thicknesses are deduced from the observed colors, compared to a color chart. The Frankel's film thickness gradient is higher is this configuration and the thickness may vary from roughly $600 \mathrm{~nm}$ close to the frontier with the thin film to $2 \mu \mathrm{m}$ close to the top meniscus. For comparison with the theory, we use the rough estimate $h_{2} \approx 1.5 \mu \mathrm{m}$.

The destabilization process is quantified using the color camera. We call $I_{1}$ the first image where oscillations are visible, and $I_{0}$ the last image before $I_{1}$. The height of the initial film and of the top Frankel's films in $I_{0}$ are called $d_{1}$ and $d_{2}$ (see Fig. 3c).

The frontier between the top Frankel's film and the initial film is automatically detected using a matlab routine (see Fig. 4). The extrema of the frontier are then identified, and the experimental wavelength $\lambda^{\exp }$ is defined as the average distance (along $x$ ) between minima.

Few minima are followed between $I_{1}$ and the following image $I_{2}$ to determine the growth rate $n$. We assume that the position along $z$ of a minimum obeys $z_{m}(t)=a$ $\exp (n t)$, with $a$ the initial noise $(a<0$ in this case) and $n$ the growth rate. We thus define the experimental value of the growth rate as

$$
n^{\exp }=\frac{1}{t_{2}-t_{1}} \ln \left(\frac{z_{m}\left(t_{2}\right)}{z_{m}\left(t_{1}\right)}\right)
$$



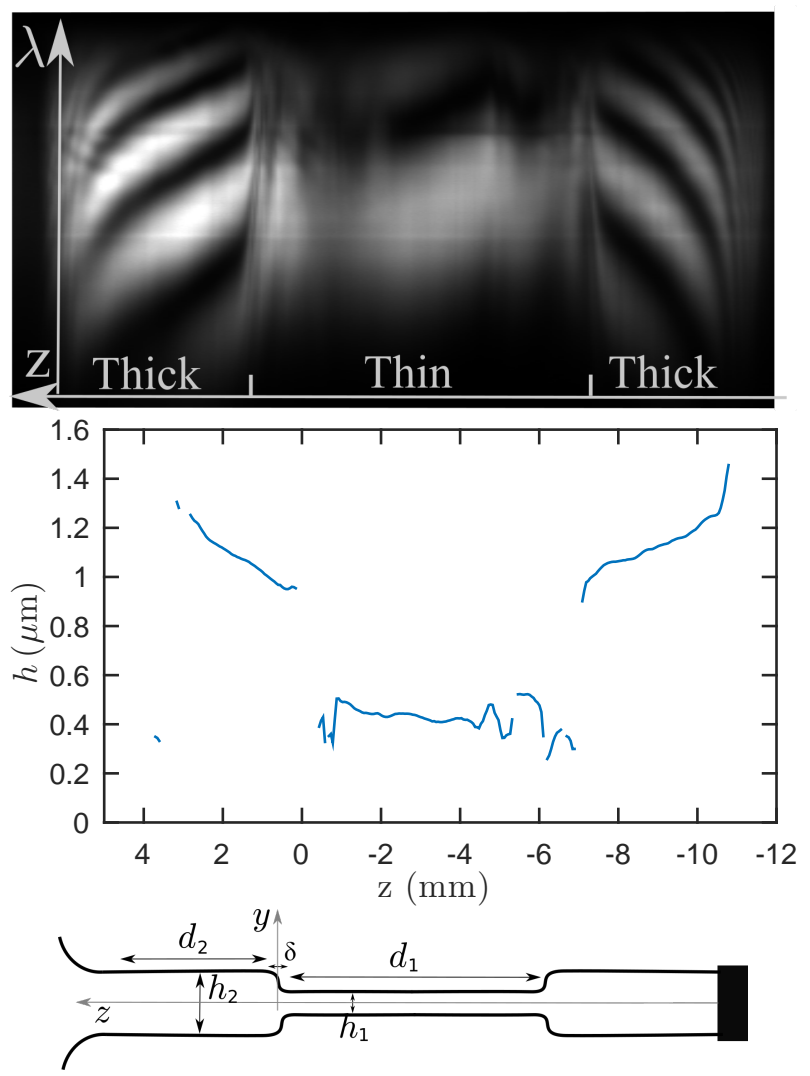

FIG. 3. Initial film thickness profile. (Top) Raw image obtained with the spectral camera, just after stretching the film. The total height in the $z$ direction is $19 \mathrm{~mm}$, and the wavelength $\lambda$ varies from $375 \mathrm{~nm}$ at the top to $1010 \mathrm{~nm}$ at the bottom. The gray level is the light intensity (in arbitrary unit). The central part corresponds to the initial thin film and the two lateral parts are the thicker parts of the film, which have been extracted from the menisci. (middle) Film thickness profile extracted from the top image. (Bottom) Sketch of the film profile and notations used in the text.

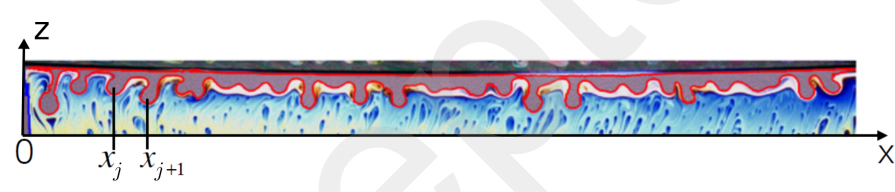

FIG. 4. Determination of the wavelength. The red line is the frontier between the initial film and the top Frankel's film, as detected by the image processing. The wavelength is the average distance between two successive minima $x_{j}$ and $x_{j+1}$ of the curve.

\section{EXPERIMENTAL RESULTS}

\section{A. Linear Regime: wavelength and growth rate}

The first series of data have been obtained with the solution $S_{a}$, on the inclined table (set-up A). In this series the film thicknesses and initial sizes are kept as con-
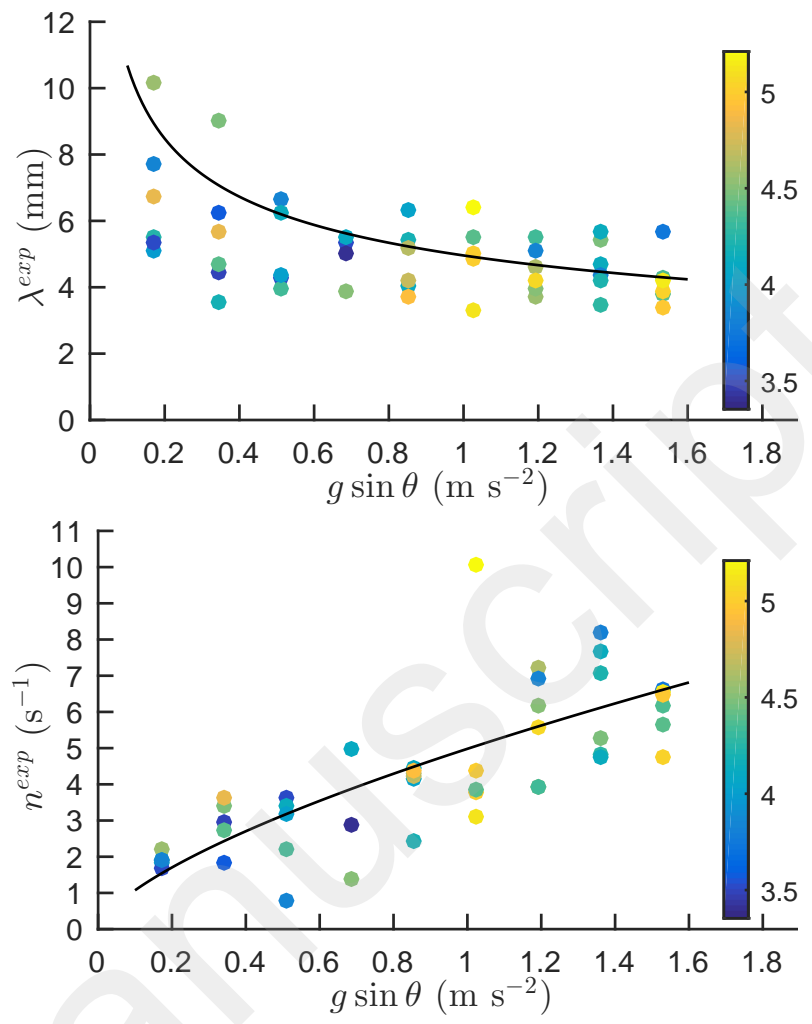

FIG. 5. Behavior in the linear regime for solution $\left(S_{a}\right)$. (Top) Wavelength as a function of the effective gravity ; (Bottom) Growth rate of the instability. The solid lines are the scaling laws given respectively by eq. 10 with the prefactor 3 (instead of the expected 19.5) and by eq. 11 with the prefactor $1 / 3.5$ (instead of the expected 1/2.8). The color indicates the value of the thick film height $d_{2}$, in $\mathrm{mm}$.

stant as possible and the effective gravity is varied. At the onset of the instability, we have $h_{1}=0.4 \pm 0.1 \mu \mathrm{m}$, $h_{2}=1.2 \pm 0.2 \mu \mathrm{m}, d_{1}=8 \pm 0.7 \mathrm{~mm}$ and $d_{2}=4.2 \pm 0.7$ $\mathrm{mm}$. The observed wavelength is shown in Fig. 5 as a function of the effective gravity $\bar{g}$ : the wavelength slightly decreases when the angle increases. The data are rather dispersed, and we checked that this variability was not correlated with the fluctuations of the system characteristics: in Fig. 5, we show that $d_{2}$, given by the color chart, is not correlated to the wavelength, at a given angle. Similarly the film thickness variations cannot explain the data dispersion.

In Fig. 5, we show that the growth rate increases with the effective gravity. Finally, as expected, the frontier between the thin and thick films is stable when the film is perfectly horizontal $(\theta=0)$.

Very similar results are obtained with the solution $S_{b}$ (with dodecanol) in almost the same conditions, as shown in Fig. 6. In that second series, $h_{1}=0.5 \pm 0.1 \mu \mathrm{m}$, $h_{2}=3 \pm 0.5 \mu \mathrm{m}$ and $d_{2}=3 \pm 0.3 \mathrm{~mm}$.

The vertical set-up (set-up B) has been used with solution $S_{b}$. In that case, the effective gravity is fixed, and we modify the characteristics of the thin and thick films 

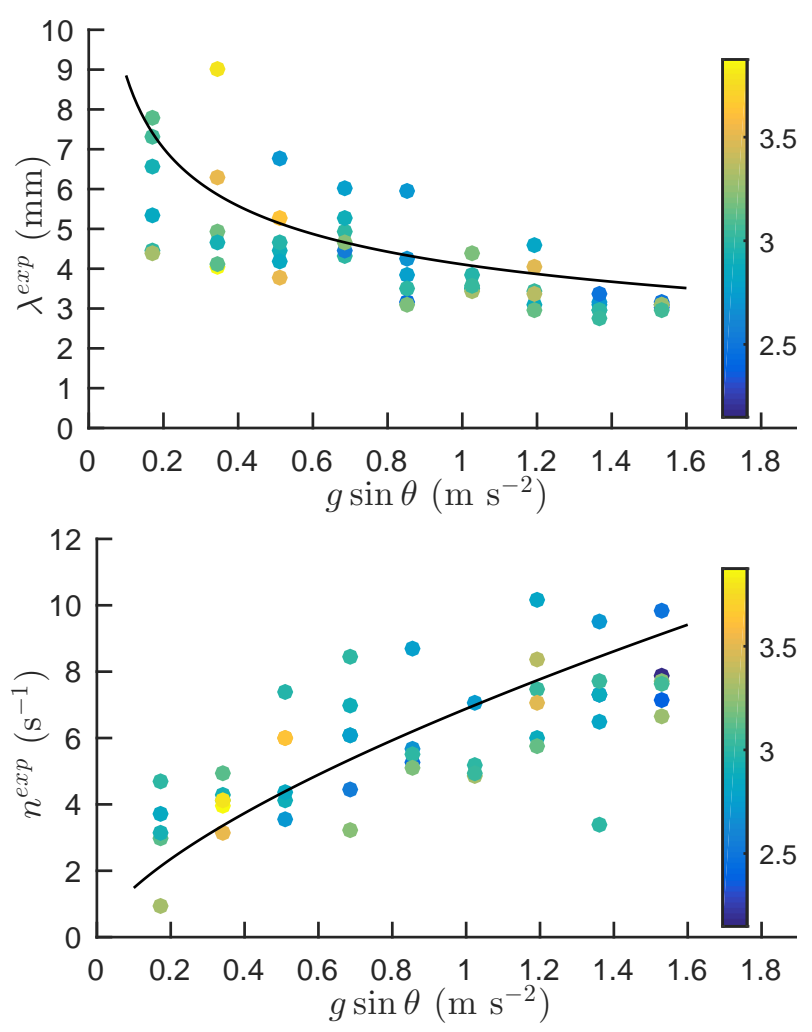

FIG. 6. Behavior in the linear regime for solution $\left(S_{b}\right)$. (Top) Wavelength as a function of the effective gravity; (Bottom) Growth rate of the instability. The solid lines are the scaling laws given respectively by eq. 10 with the prefactor 4.5 (instead of the expected 19.5) and by eq. 11 with the prefactor $1 / 4$ (instead of the expected $1 / 2.8$ ). The color indicates the value of the thick film height $d_{2}$, in $\mathrm{mm}$.

by changing the motor velocity from $U_{m}=5 \mathrm{~mm} / \mathrm{s}$ to the maximal rate $U_{m}=112 \mathrm{~mm} / \mathrm{s}$. As the stretching of the initial film and the extraction of the Frankel's film are two competing effects occurring simultaneously during and after linear stage motion, the various geometrical parameters $h_{1}, h_{2}, d_{1}$ and $d_{2}$ can not be varied independently.

The obtained parameter range is $[0.2-1.2] \mathrm{mm}$ for $d_{2}$ and $[0.3-0.7] \mu \mathrm{m}$ for $h_{1}$. We only have an estimation for $h_{2}$, which is in the range $1-2 \mu \mathrm{m}$. The thin film height is always much larger than $d_{2}$ and plays no role. Fig. 7 shows the wavelength and the growth rate as a function of the thick film height $d_{2}$. The color indicates the thin film thickness $h_{1}$, with an error bar of $100 \mathrm{~nm}$.

These experimental data are discussed in section $\mathrm{V}$, and compared to the model developed below.
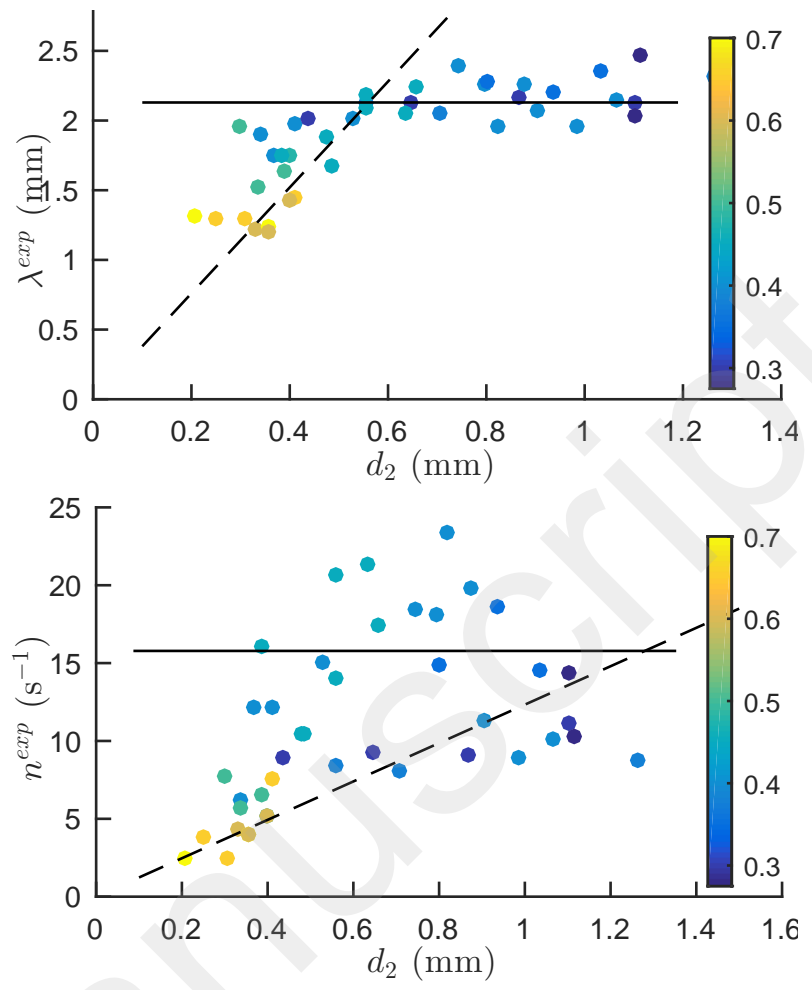

FIG. 7. Experimental wavelength (top) and growth rate (bottom) as a function of $d_{2}$, for solution $S_{b}$ with the vertical set-up. The thin film thickness $h_{1}$, expressed in $\mu \mathrm{m}$, is given by the color chart. (Top) dashed line: scaling law 12; solid line: scaling law 10 with the prefactor 3.12 (instead of the expected 19.5). (Bottom) dashed line: scaling law 13; solid line: scaling law 11 with the prefactor $1 / 5.6$ (instead of the expected $1 / 2.8)$.

\section{THEORY: LINEAR STABILITY ANALYSIS}

\section{A. Model assumptions}

The Frankel's film extracted at the bottom is stable. It plays no role in the instability and will not be taken into account in the model, which will focus on the coupling between the thick film at the top, of thickness $h_{2} \approx 2 \mu \mathrm{m}$ and height $d_{2}$ (region 2 of the film) and the thin film at the bottom, of thickness $h_{1} \approx 0.5 \mu \mathrm{m}$ and height $d_{1}$ (region 1 ). The thicknesses are always much larger than 100 $\mathrm{nm}$ and the disjoining pressure is thus negligible. The fluctuations of $h_{1}$ or $h_{2}$ with time and space are much smaller than the thickness difference $h_{2}-h_{1}$, so a uniform thickness will be assumed in each region. The transition between one region to the other occurs on a typical length $\delta$ of the order of $100 \mu \mathrm{m}$ (see Fig. 3 and [17]). This length is smaller than the observed wavelength and a sharp transition is therefore assumed between both regions. The frontier is located along the line $z=d(x, t)$ which will be predicted by the model.

The fluid dynamics is governed by the lubrication 
equations and the velocity and pressure fields $\mathbf{V}$ and $P$ thus obey, in each part of the film,

$$
\mathbf{V}_{i}=\frac{1}{\eta}\left(y-\frac{h_{i}}{2}\right)^{2}(\nabla P+\rho \mathbf{g})+\mathbf{v}_{i}
$$

with $\mathbf{v}_{i}$ the interfacial velocity in the domain $i$. The pressure gradients $\nabla P$ are governed by the Laplace pressure and are localized at the frontier between both films, where the interface curvature is non zero. They scale as $\gamma\left(h_{2}-h_{1}\right) / \delta^{3} \sim 10^{4} \mathrm{~m}^{2} / \mathrm{s}$, and are thus comparable to the gravity term $\rho g$ in the frontier vicinity. The induced Poiseuille flow occurring in the film even before the instability begins to develop) scales as $(\rho g+\nabla P) h^{2} / \eta \sim$ $10 \mu \mathrm{m} / \mathrm{s}$, whereas the observed velocities are of the order of $10 \mathrm{~mm} / \mathrm{s}$ : the velocities are thus dominated by the interfacial velocities $\mathbf{v}_{i}$. In the following, we neglect this Poiseuille contribution and assume that the velocity is uniform across the film thickness and is equal to $\mathbf{v}_{i}(x, z, t)$, and that the pressure is homogeneous and equal to the air pressure. One important consequence is that an elementary piece of film of volume $h_{i} d x d z$, taken either in the thin or in the thick film, is a closed material system. Especially, there is no liquid flux across the frontier $d$ between both film regions.

We assume that the instability can be described using an inextensible interface model. This assumption implies that each piece of film $d x d z$ keeps a constant thickness with time.

Finally, the instability begins to grow during the Frankel's film extraction, so the reference state, characterized by $d_{1}$ and $d_{2}$, depends on time. However, in the following, we make the crude assumption that the instability time scale is much smaller than the extraction time scale and that $d_{1}$ and $d_{2}$ can be assumed to be constant. To summarize, the main physical processes can be reproduced assuming that the parts of film (1) and (2) are of constant and uniform thickness $h_{1}$ and $h_{2}$, and of constant area $w d_{1}$ and $w d_{2}$.

The evolution of the frontier is governed by the gravity $\bar{g}$, the surface tension of the film $\sigma$ (taking into account both interfaces) and the shear viscosity of the film $\mu_{s, i}=$ $\eta h_{i}+2 \eta_{s}$. The interface viscosity is typically of the order of $\eta_{s} \approx 10^{-6} \mathrm{~kg} \mathrm{~s}^{-1}$ [23], much larger than $\eta h \sim 10^{-9} \mathrm{~kg}$ $\mathrm{s}^{-1}$. The film shear viscosity can thus be approximated by $\mu_{s}=2 \eta_{s}$ in both parts of the film.

\section{B. Reference state}

In the reference state, the frontier $d_{0}(x)$ between parts (1) and (2) of the film is a horizontal straight line and the reference for $z$ is chosen so that $d_{0}(x)=0$. The interface velocities are vanishing and the whole system is at rest. As the Laplace and disjoining pressures are negligible in the film, the pressure in the liquid phase is the atmospheric pressure, taken as a reference pressure. The force balance in the $z$ direction on a piece of film $d x d z$ therefore implies only the tension and the gravity:

$$
0=\frac{\partial \sigma}{\partial z}-\rho h \bar{g}
$$

We thus get

$$
\sigma_{1}^{0}=\rho \bar{g} h_{1} z+2 \gamma_{0} \quad \text { and } \quad \sigma_{2}^{0}=\rho \bar{g} h_{2} z+2 \gamma_{0}
$$

in the reference state, with $\gamma_{0}$ the interface tension reference, taken arbitrary at $z=0$. It is analog to a hydrostatic pressure in a $3 \mathrm{D}$ problem.

\section{Scaling laws}

In order to determine the stability of the reference state, a frontier shape $d(x, t)=\varepsilon e^{n t} e^{i k x}$ is assumed. Before dealing with the full linear stability analysis we first derive scaling laws for $n(k)$ based on simple force balances.

We first deal with the system (1) shown in Fig. 8(left), made of piece of film of size $\lambda \times \lambda$ across the frontier, with $\lambda=2 \pi / k$ the perturbation wavelength.

In each film, all horizontal oscillations of the physical quantities damp exponentially with $z$ over a distance comparable to the wavelength. For this scaling law analysis, let us boldly assume that they are independent of $x$ at $z= \pm \lambda, i$. e. at the top and bottom boundaries of system (1).
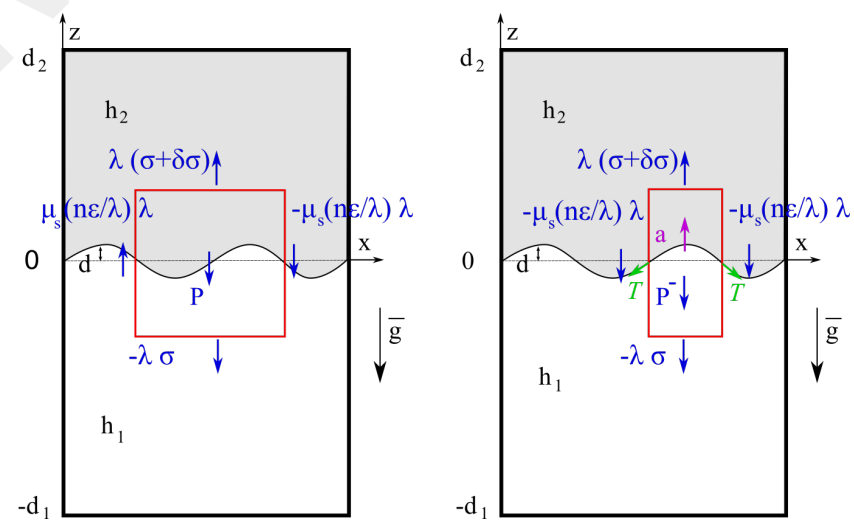

FIG. 8. Various forces acting on system (1) and (2), limited by the red rectangles respectively in the left and right schemes. (Left) The force balance on the system (1) involves surface tension and gravity, as viscous forces compensate on both sides. (Right) On the system (2), gravity force is reduced by a larger amount of thin film, and interfacial viscous forces and inertia need to be considered.

The surface tension is denoted by $\sigma+\Delta \sigma$ at the top boundary and $\sigma$ at the bottom.

The mass of the system is (disregarding any numerical prefactor) $m=\lambda^{2} \rho\left(h_{1}+h_{2}\right)$. The acceleration is vanishing by symmetry: the left part moves down and the right part moves up. Similarly, periodicity implies that 
the viscous forces on both sides compensate each other. Finally, the resulting force due to the surface tension is $\lambda \Delta \sigma \mathbf{e}_{z}$ and the weight is $-\lambda^{2} \rho \bar{g}\left(h_{1}+h_{2}\right) \mathbf{e}_{z}$. The force balance on the system thus imposes

$$
\Delta \sigma \sim \lambda \rho \bar{g}\left(h_{1}+h_{2}\right) .
$$

The equilibrium relation (4) thus remains true in the out of equilibrium case, far enough from the frontier.

We now focus on the subsystem (2), made of the right part of the previous system, moving upwards (see Fig. 8 (right)). The surface tension contribution is still $F_{\sigma}=\lambda \Delta \sigma$, but it now slightly overcompensates the gravity $F_{g}=-\lambda \rho \bar{g}\left[h_{1}(\lambda+\varepsilon)+h_{2}(\lambda-\varepsilon)\right]$. Using eq. (6), the resulting force can be expressed as $F_{\sigma}+F_{g}=\lambda \rho \bar{g} \varepsilon\left(h_{2}-h_{1}\right)$.

One damping term is the film inertia, scaling as $\lambda^{2} \rho\left(h_{1}+h_{2}\right) n^{2} \varepsilon$. Another arises from the interfacial viscous forces: on both lateral sides of the system (length of order $\lambda$ ), the velocity gradients scale as $n \varepsilon / \lambda$ (see Fig. 8 (right)) and the resulting force thus scales as $-\mu_{s} n \varepsilon$.

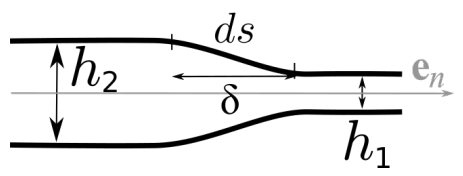

FIG. 9. Origin of the line tension. We consider the frontier width, measured in the plane normal to the frontier: the width $d s$ measured along the interface shape is slightly larger than the width $\delta$ measured in the film plane. It induces a resulting force $T$ oriented along the frontier tangent.

From the thickness profiles, we can define a line tension $T$ between the domains (1) and (2) (see Fig. 9). Its order of magnitude can be determined from the area excess induced by the frontier. As the thickness varies from $h_{1}$ to $h_{2}$ over a distance $\delta$ perpendicular to a local line element $d \ell$, the area of this transition region is $a_{\text {int }}=$ $d \ell\left(\delta^{2}+\left(h_{2}-h_{1}\right)^{2}\right)^{1 / 2} \sim d \ell \delta\left(1+\left(h_{2}-h_{1}\right)^{2} /\left(2 \delta^{2}\right)\right)$. The excess area due to the thickness variation is thus $\left(h_{2}-\right.$ $\left.h_{1}\right)^{2} /(2 \delta)$ per unit length. The resulting line tension $T$ scales as $\sigma\left(h_{2}-h_{1}\right)^{2} / \delta \sim 10^{-10} \mathrm{~N}$, and the associated force on the system includes the slope $\varepsilon / \lambda$ and scales as $-\varepsilon \sigma\left(h_{2}-h_{1}\right)^{2} /(\lambda \delta)$ (see Fig. 8(right)).

This line tension term dominates at very small wavelength. Balancing the gravity excess and the line tension, we show that it ensures the stabilization of perturbations having a wavelength smaller than $\lambda_{c}$, with

$$
\lambda_{c}=\left[\frac{\sigma\left(h_{2}-h_{1}\right)}{\rho \bar{g} \delta}\right]^{1 / 2} .
$$

All length scales smaller than this cut-off are stable. At small angles, $\lambda_{c}$ is in the range $500 \mu \mathrm{m}-1 \mathrm{~mm}$ for $\bar{g}$ between 0.2 and $1.6 \mathrm{~m}^{2} / \mathrm{s}$, and for the vertical case $\lambda_{c} \sim 200 \mu \mathrm{m}$. In both cases it is 5 times smaller than the observed wavelengths. Note that for $\lambda \sim \delta \sim 100 \mu \mathrm{m}$, the model of sharp frontier is not valid anymore, and a more refined model would be needed in order to take the tension into account in a relevant way.

The damping induced by the friction on air can also become relevant, as it is the case for giant films [24]. Assuming that the air in contact with the film is at rest at the instability onset, we can estimate its contribution. The thickness of the laminar visco-inertial boundary layer in air scales as $\delta_{a}=\left[\mu_{a} /\left(\rho_{a} n\right)\right]^{1 / 2} \sim 1 \mathrm{~mm}$ and the induced friction on the system is $F_{f} \sim \lambda^{2} \mu_{a} n \varepsilon / \delta_{a}$. The ratio between this viscous force and the inertial term is $\rho_{a} \delta_{a} /(\rho h)$, as expected by considering the boundary layer in air as an added mass to the system. This ratio remains slightly lower than 1 for our experimental values of the parameters.

Finally, in this first attempt to rationalize our observations with a semi-analytical model, we chose to keep it as simple as possible and to neglect the line tension and the friction on air. We consequently keep only two damping terms, the interfacial viscous forces and the inertia, scaling respectively as $\lambda^{0}$ and $\lambda^{2}$ to balance the driving force, which is the excess of gravity force, scaling as $\lambda^{1}$. The potential influence of the neglected terms is discussed qualitatively in section $\mathrm{V}$.

Just above $\lambda_{c}$, the instability is damped by the viscosity and the growth rate is obtained by balancing gravity and interfacial viscosity terms

$$
n_{v i s q} \sim \frac{\left(h_{2}-h_{1}\right) \lambda \rho \bar{g}}{\mu_{s}} .
$$

Similarly, in the large $\lambda$ limit, the damping term is the inertia and the growth rate scales as

$$
n_{\text {inert }}=\left(\frac{\left(h_{2}-h_{1}\right) \bar{g}}{\left(h_{1}+h_{2}\right) \lambda}\right)^{1 / 2} .
$$

The full numerical prediction, based on the approximations discussed above and on the equations established in the next paragraph, is shown in Fig. 10. The power laws given by eq. (8) and (9) are recovered at small and large $\lambda$, respectively. There is a direct parallel with the usual Rayleigh - Taylor instability in 3D systems, surface tension playing the role of the pressure and film thickness replacing the density.

The fastest growth rate $n_{t h, 1}$ is obtained for the wavelength $\lambda_{t h, 1}$ verifying $n_{v i s q} \sim n_{\text {inert }}$. Using the prefactors obtained by comparison with the numerical prediction (see Fig. 11), we get

$$
\lambda_{t h, 1} \simeq 19.5\left(\frac{\mu_{s}^{2}}{\left(h_{2}^{2}-h_{1}^{2}\right) \rho^{2} \bar{g}}\right)^{1 / 3}
$$

and

$$
n_{t h, 1} \simeq \frac{1}{2.8}\left(\frac{\left(h_{2}-h_{1}\right)^{2} \bar{g}^{2} \rho}{\left(h_{2}+h_{1}\right) \mu_{s}}\right)^{1 / 3}
$$




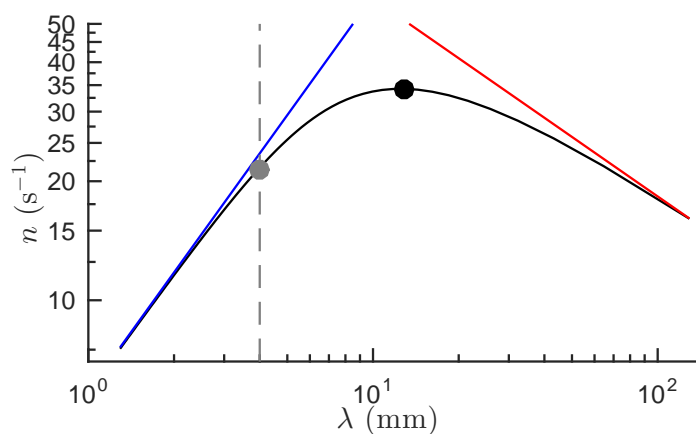

FIG. 10. Value of $n$ as a function of the wavelength with $\rho=$ $10^{3} \mathrm{~kg} / \mathrm{m}^{-3}, h_{1}=0.510^{-6} \mathrm{~m}, h_{2}=210^{-6} \mathrm{~m}, \mu_{s}=10^{-7} \mathrm{~kg} / \mathrm{s}$ and $\bar{g}=9.8$. Black solid line: solution of the equation set (eqs. (25)-(27), (30) and (35)-(36)); blue and red line: asymptotic scaling laws given by eq. (8) and (9), respectively. The fastest mode is represented by the black dot, close to the intersection of both asymptotes. The gray dashed line is at the abscissa $d_{2}$ and the large wavelengths at the right of this line are forbidden. If this cut off at $d_{2}$ occurs at a wavelength smaller than the fastest one, than the gray dot represents the actual fastest mode.

For $\mu_{s}=10^{-7} \mathrm{~kg} \cdot \mathrm{s}^{-1}, h_{2}=2 \mu \mathrm{m}, h_{1}=0.5 \mu \mathrm{m}$ we find $\lambda_{t h, 1}$ of the order of few millimeters, which may be comparable to the thick film height $d_{2}$. We thus expect a cut-off at $d_{2}$ of the scaling for $\lambda$ : wavelengths larger than $d_{2}$ can not grow, so if $\lambda_{t h, 1}>d_{2}$ then the fastest wavelength scales as $d_{2}$ and its growth rate is controlled by eq. (8) (see Fig. 10). This imposes, for the cases $\lambda_{t h, 1}>d_{2}$,

$$
\lambda_{t h, 2} \simeq 3.8 d_{2},
$$

and

$$
n_{t h, 2} \simeq \frac{1}{10.5} \frac{\left(h_{2}-h_{1}\right) d_{2} \rho \bar{g}}{\mu_{s}} .
$$

Here again, the prefactors result from fits of the numerical solutions (see Fig. 11).

\section{Equations in the bulk}

These scaling laws clearly identify the physical processes, which are modeled below. The stability analysis of the usual Rayleigh-Taylor instability have been done for $2 \mathrm{D}$ systems in [25]. We re-establish the dispersion relation here, in the context of soap films, and extend it to finite size systems.

We define the interface velocity $\mathbf{v}_{i}=\left(u_{i}, w_{i}\right)$ and the film tension $\sigma=\sigma^{0}+\delta \sigma$, with $\sigma^{0}$ given by eq. (5).

The assumption of inextensible interfaces leads to

$$
\partial_{x} u+\partial_{z} w=0
$$

The force balance written on a film piece $d x d z$ of thickness $h$ (of value either $h_{1}$ or $h_{2}$ ) is, projected in the $x$ and
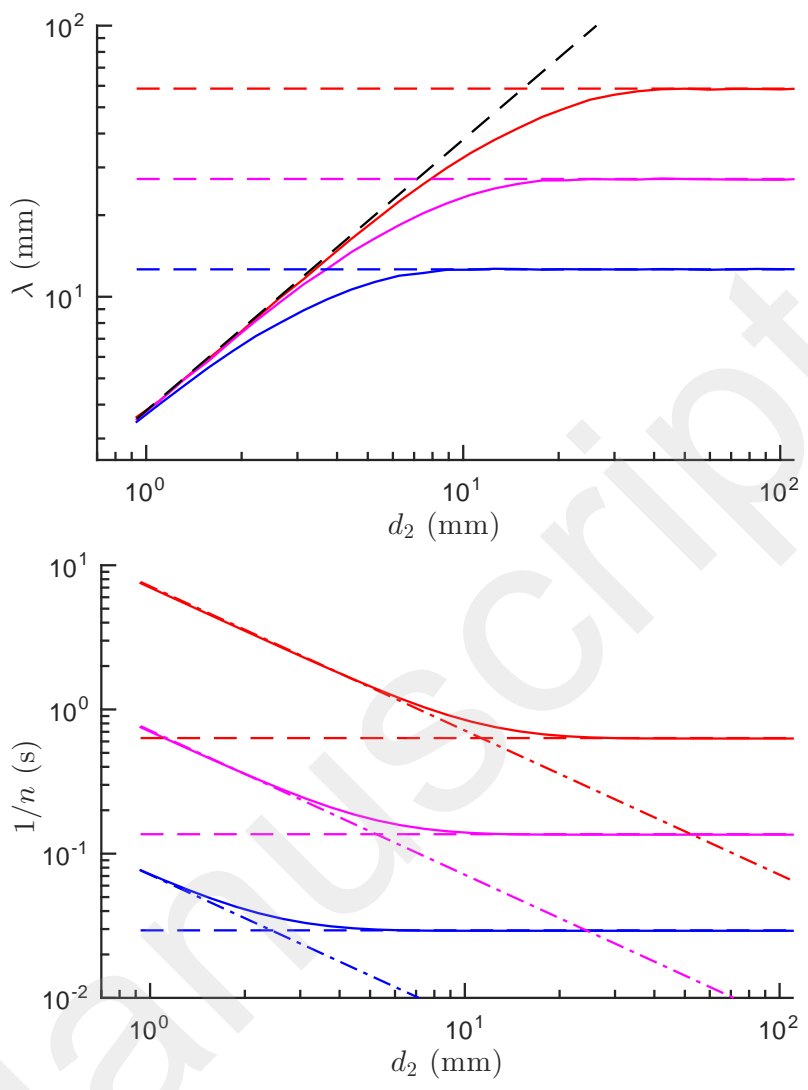

FIG. 11. Theoretical fastest wavelength (top) and associated time scale (bottom) as a function of the upper film size $d_{2}$. The parameters are $h_{1}=0.5 \mu \mathrm{m}, h_{2}=2 \mu \mathrm{m}, \mu_{s}=10^{-7} \mathrm{~kg} \cdot \mathrm{s}^{-1}$, and an infinite value of $d_{1}$. The angle $\theta$, governing the apparent gravity, verifies $\sin \theta=0.01,0.1,1$ respectively for the red, magenta and blue lines. (Top) Horizontal dashed lines: $19.5 \lambda_{t h, 1}$ (eq. (10)); black dashed line: $3.8 \lambda_{t h, 2}$ (eq. (12)). (Bottom) Dashed lines: $2.8 / n_{t h 1}$ (eq. (11)) ; dot-dashed lines: $10.5 / n_{t h 2}$ (eq. (13)). Solid lines are the numerical solutions $\lambda^{\text {num }}$ and $n^{\text {num }}$ of the full system (eqs. (25)-(27), (30) and (35)-(36)). The prefactors of the scaling laws eqs. (10)-(13) are fitted on these numerical solutions.

$z$ directions

$$
\begin{aligned}
& \rho h\left(\partial_{t} u+u \partial_{x} u+w \partial_{z} u\right)= \\
& \partial_{x} \sigma+\mu_{s}\left(\partial_{x x} u+\partial_{z z} u\right) \\
& \rho h\left(\partial_{t} w+u \partial_{x} w+w \partial_{z} w\right)= \\
& \partial_{z} \sigma+\mu_{s}\left(\partial_{x x} w+\partial_{z z} w\right)-\rho \bar{g} h .
\end{aligned}
$$

At leading order (ie, order 0 in $\varepsilon$ ) we recover the equilibrium equation for the reference state eq. (4) and, at order 1 in $\varepsilon$, eqs. (15), (16) and (14) become respectively:

$$
\begin{aligned}
& \rho h n u=i k \delta \sigma+\mu_{s}\left(-k^{2} u+\partial_{z z} u\right), \\
& \rho h n w=\partial_{z} \delta \sigma+\mu_{s}\left(-k^{2} w+\partial_{z z} w\right), \\
& i k u=-\partial_{z} w .
\end{aligned}
$$

Substituting $\delta \sigma$ and $u$ in eqs (17)-(19), we get an equa- 
tion on $w$ only:

$$
\mu_{s} \partial_{4 z} w-\left(\rho h n+2 k^{2} \mu_{s}\right) \partial_{z z} w+\left(\mu_{s} k^{4}+\rho h n k^{2}\right) w=0,
$$

whose solutions are, in domains $j=1$ or 2 ,

$$
w_{j}=A_{j} e^{k z}+B_{j} e^{-k z}+C_{j} e^{q_{j} z}+D_{j} e^{-q_{j} z},
$$

with

$$
q_{j}=\left(\frac{\rho h_{j} n}{\mu_{s}}+k^{2}\right)^{1 / 2}
$$

and $A_{j}, B_{j}, C_{j}$ and $D_{j}$ coefficients we need to determine from the boundary conditions.

The x-component of the velocity and the dynamical surface tension in each domain can be expressed as a function of $w$ from eqs. (17) and (19):

$$
\begin{aligned}
& u=\frac{i}{k} \partial_{z} w \\
& \delta \sigma=\frac{\rho h n}{k^{2}} \partial_{z} w+\frac{\mu_{s}}{k^{2}}\left(k^{2}-\partial_{z z}\right) \partial_{z} w .
\end{aligned}
$$

The second relation is obtained by substituting eq. (23) in eq. (17).

\section{E. Boundary condition at the interface}

The normal velocity continuity at the frontier $w_{1}(d)=$ $w_{2}(d)$ leads to:

$$
A_{1}+B_{1}+C_{1}+D_{1}=A_{2}+B_{2}+C_{2}+D_{2},
$$

and the tangential velocity continuity $u_{1}(d)=u_{2}(d)$ provides, using eq.(23):

$$
\begin{aligned}
& k A_{1}-k B_{1}+q_{1} C_{1}-q_{1} D_{1}= \\
& k A_{2}-k B_{2}+q_{2} C_{2}-q_{2} D_{2}
\end{aligned}
$$

The continuity of the tangential stress $\mu_{s}\left(\partial_{z} u_{1}+\partial_{x} w_{1}\right)=\mu_{s}\left(\partial_{z} u_{2}+\partial_{x} w_{2}\right)$ is :

$$
\begin{aligned}
& 2 k^{2}\left(A_{1}+B_{1}\right)+\left(q_{1}^{2}+k^{2}\right)\left(C_{1}+D_{1}\right)= \\
& 2 k^{2}\left(A_{2}+B_{2}\right)+\left(q_{2}^{2}+k^{2}\right)\left(C_{2}+D_{2}\right)
\end{aligned}
$$

Finally, disregarding the role of the line tension, the continuity of the normal stress imposes

$$
\begin{aligned}
& 2 \mu_{s} \partial_{z} w_{1}+\partial_{z} \sigma_{1} \varepsilon+\delta \sigma_{1}= \\
& 2 \mu_{s} \partial_{z} w_{2}+\partial_{z} \sigma_{2} \varepsilon+\delta \sigma_{2} .
\end{aligned}
$$

This expression involves $\varepsilon$ because $\partial_{z} \sigma$ is of order 0 . Its value is obtained from the kinematic condition $\partial_{t} d=$ $w_{1}=w_{2}$ :

$$
n \varepsilon=w_{1} .
$$

The value of $\delta \sigma$ is given by eq. (24). After some reorganization proposed in [25] and given in Appendix 1, the condition becomes

$$
\begin{aligned}
& 0=A_{1}\left(\frac{R}{2}-\alpha_{1}\right)+B_{1}\left(\frac{R}{2}+\alpha_{1}\right)+C_{1} \frac{R}{2}+D_{1} \frac{R}{2}+ \\
& A_{2}\left(\frac{R}{2}+\alpha_{2}\right)+B_{2}\left(\frac{R}{2}-\alpha_{2}\right)+C_{2} \frac{R}{2}+D_{2} \frac{R}{2},
\end{aligned}
$$

with

$$
\begin{aligned}
R & =\frac{\bar{g} k}{n^{2}} \frac{h_{2}-h_{1}}{h_{1}+h_{2}}, \\
\alpha_{i} & =\frac{h_{i}}{h_{1}+h_{2}} .
\end{aligned}
$$

\section{F. Limit of large $d_{1}$ and $d_{2}$}

The velocity remains finite so $A_{2}=0, B_{1}=0, C_{2}=0$, $D_{1}=0$. The system is then

$$
\begin{aligned}
& A_{1}+C_{1}-B_{2}-D_{2}=0, \\
& k A_{1}+q_{1} C_{1}+k B_{2}+q_{2} D_{2}=0, \\
& 2 k^{2} A_{1}+\left(q_{1}^{2}+k^{2}\right) C_{1}-2 k^{2} B_{2}-\left(q_{2}^{2}+k^{2}\right) D_{2}=0, \\
& A_{1}\left(R-2 \alpha_{1}\right)+C_{1} R+B_{2}\left(R-2 \alpha_{2}\right)+D_{2} R=0,
\end{aligned}
$$

in agreement with the system eq. (111) in [25], for a problem in bulk, without surface tension.

This system has non trivial solutions only if its determinant is zero, which imposes, as established in Appendix 2 ,

$$
0=(R-1)\left(q_{1} \alpha_{2}+q_{2} \alpha_{1}-k\right)-4 k \alpha_{1} \alpha_{2} .
$$

This is eq. 113 in [25].

With the parameters of Fig. 10, the model predicts that the most unstable wavelength is $1.5 \mathrm{~cm}$, to be compared with the $3 \mathrm{~mm}$ experimentally observed. As already seen with the scaling laws, we have to consider the finite size of the system: there is a cut-off on the wavelength when the height of one region $\left(d_{1}\right.$ or $\left.d_{2}\right)$ is too small.

\section{G. Finite value of $d_{2}$}

For finite size systems, conditions must be imposed at the positions $d_{1}$ and $d_{2}$. As we have $d_{1} \gg d_{2}$, we only consider the finite value of $d_{2}$. The precise condition at the boundary with the free meniscus is actually a complicated problem. The Laplace pressure becomes important close to the meniscus and the equations of motion used here fail. For sake of simplicity, we simply impose vanishing tangential and normal velocities. This leads to, at $z=d_{2}$ :

$$
\begin{aligned}
& A_{2} e^{k d_{2}}+B_{2} e^{-k d_{2}}+C_{2} e^{q_{2} d_{2}}+D_{2} e^{-q_{2} d_{2}}=0 \\
& k A_{2} e^{k d_{2}}-k B_{2} e^{-k d_{2}}+q_{2} C_{2} e^{q_{2} d_{2}}-q_{2} D_{2} e^{-q_{2} d_{2}}=0
\end{aligned}
$$




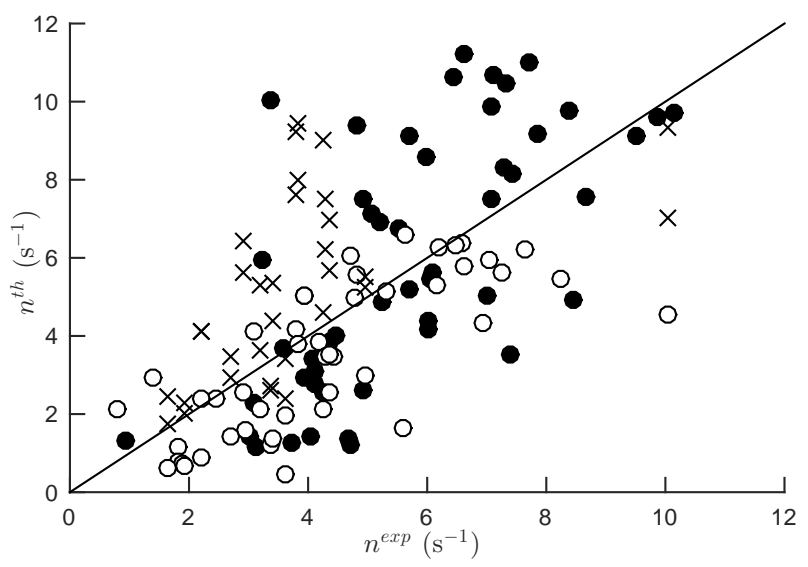

FIG. 12. Theoretical growth rate as a function of the experimental value shown in Fig. $5(\circ), 6(\bullet)$ and $7(\times)$. The theoretical value is determined by numerical resolution of the system, using the experimental values of $d_{2}, h_{2}$ and $h_{1}$ (we used $h_{2}=1.5 \mu \mathrm{m}$ for the vertical series). The other parameters are $\rho=10^{3} \mathrm{~kg} / \mathrm{m}^{3}$ and $\mu_{s}=0.810^{-7} \mathrm{~kg} / \mathrm{s}$. The line corresponds to $n^{\text {th }}=n^{\text {exp }}$.

We thus obtain a system with 6 equations and 6 unknown eqs. (25)-(27), (30) and (35)-(36). For a given set of physical parameters, and for a suited range of $k$, we determine numerically the value of $n(k)$ for which the system determinant vanishes. We adapt the range of $k$ to make sure that $n(k)$ has a maximum $n_{\max }\left(k_{\max }\right)$ in that range. We solved the problem for the parameter sets $h_{1}=0.5 \mu \mathrm{m}, h_{2}=2 \mu \mathrm{m}, \mu_{s}=10^{-7} \mathrm{~kg} \cdot \mathrm{s}^{-1}$, for 3 values of the effective gravity, and for $d_{2}$ between 1 and 100 mm. The corresponding wavelengths $\lambda=2 \pi / k_{\max }$ and characteristic times $\tau=1 / n_{\max }$ are shown in Fig. 11: as expected, the scaling laws previously established are recovered in the limit of small and large $d_{2}$.

\section{COMPARISON WITH THE EXPERIMENTAL DATA}

To compare our predictions with the experimental data, we took into account in the model, for each experimental data, the measured value of the parameters $h_{1}, h_{2}, d_{2}, \bar{g}$. For the set up (A), the only unknown parameter is the interfacial shear viscosity $\mu_{s}$. For set-up (B) (without spectral camera), the thickness $h_{1}$ has been deduced from the comparison of the foam film color and a reference color chart with an uncertainty of $\pm 100 \mathrm{~nm}$. The error bar on $h_{2}$ is larger, but counting the interference fringes indicates a value in the range $[1-2] \mu \mathrm{m}$. The thickness $h_{2}=1.5 \mu \mathrm{m}$ has been used in the model. The comparison between the growth rates obtained theoretically and experimentally is shown in Fig. 12 and shows a reasonable agreement for $\mu_{s}=0.810^{-7} \mathrm{~kg} \mathrm{~s}^{-1}$, for both solutions. The order of magnitude of this viscosity is the expected one for the SDS solution. Indeed $\eta_{s}=\mu_{s} / 2=0.6 \pm 0.410^{-7} \mathrm{~kg} \cdot \mathrm{s}^{-1}$ has been found in [23]. A slightly larger value was expected for the solution with dodecanol.

Then, using $\mu_{s}=0.810^{-7} \mathrm{~kg} . \mathrm{s}^{-1}$, the experimental data of Figs. 5, 6 and 7 can be compared to the scaling laws eqs.(10), (11) at large $d_{2}$ (solid lines in the figures) and eqs. (12), (13) at small $d_{2}$ (dashed lines in the figures). In Fig. 5 and 6 , we observe no correlation between $d_{2}$ and $\lambda$, which indicates that the large $d_{2}$ scaling should be used. Consistently, we show in Fig. 5 and 6 that the variations of $\lambda$ and $n$ are compatible respectively with the scalings $g^{-1 / 3}$ (eq. (10)) and $g^{2 / 3}$ (eq. (11)). Similarly, the wavelengths of Fig. 7 are compatible with a scaling as $d_{2}$ (eq. (12)) at small $d_{2}$ and a constant value (eq. (10)) at large $d_{2}$.

However, the prefactors used for these fits differs from the predicted ones. Especially the power law (10) is predicted to have a prefactor of the order of 20 , whereas a prefactor of the order of 4 is systematically needed to fit the data of Figs. 5, 6 and 7. As $\lambda$ is of the order of 2 or 3 times $d_{2}$, the finite size effect should be non negligible and the measured value for $\lambda$ should be in contrast smaller than the asymptotic prediction of eq. (10), as shown in Fig. 11.

Finally all the measured wavelengths are plotted as a function of the full numerical prediction in Fig. 13. It confirms that the model is not fully quantitative and that the transition from the large to the small film height regime is not entirely understood.

As the predicted exponents of the film thicknesses and of the interfacial viscosity are small, the discrepancy can not be attributed to the uncertainty on these quantities. The line tension stabilizes the short wavelengths, and taking it into account would lead to a larger theoretical fastest wavelength, thus increasing the disagreement with the model. The friction on air, in contrast, would decrease the growth rate in eq. (9) and would thus lead to a smaller theoretical wavelength in Fig. 10. Another possible reason for the overestimation of the wavelength by the model is that the system is not steady. The thick film height $d_{2}$ varies during the instability development and the observed wavelength may thus be the fastest mode associated to an earlier value of $d_{2}$. As $\lambda^{t h}$ increases with $d_{2}$, this effect is compatible with the observed overestimation. Finally, the boundary condition at the top used in the model, i.e. a vanishing velocity, may be oversimplified. The normal velocity at the top meniscus must be small, but the tangential velocity at the meniscus may be non negligible. A part of the meniscus is indeed set into motion in the $x$ direction, by viscous friction on the film. The typical length $\delta_{m}$ of this domain can be determined from the balance between its inertia $\rho h \delta_{m} n u$ and the viscous force $\mu_{s} u / \delta_{m}^{2}$. This leads to $\delta_{m} \sim\left(\mu_{s} /\left(\rho h \delta_{m} n\right)\right)^{1 / 2}$. At the distance $\delta_{m}$ from the film, the thickness scales as $\delta_{m}^{2} / r_{m}$ (with $r_{m}$ the meniscus radius) so we obtain the power law $\delta_{m} \sim\left(\mu_{s} r_{m} /(\rho n)\right)^{1 / 4} \sim 300 \mu \mathrm{m}$. A more physical tangential boundary condition, expressing the tangential stress continuity at the frontier between the 

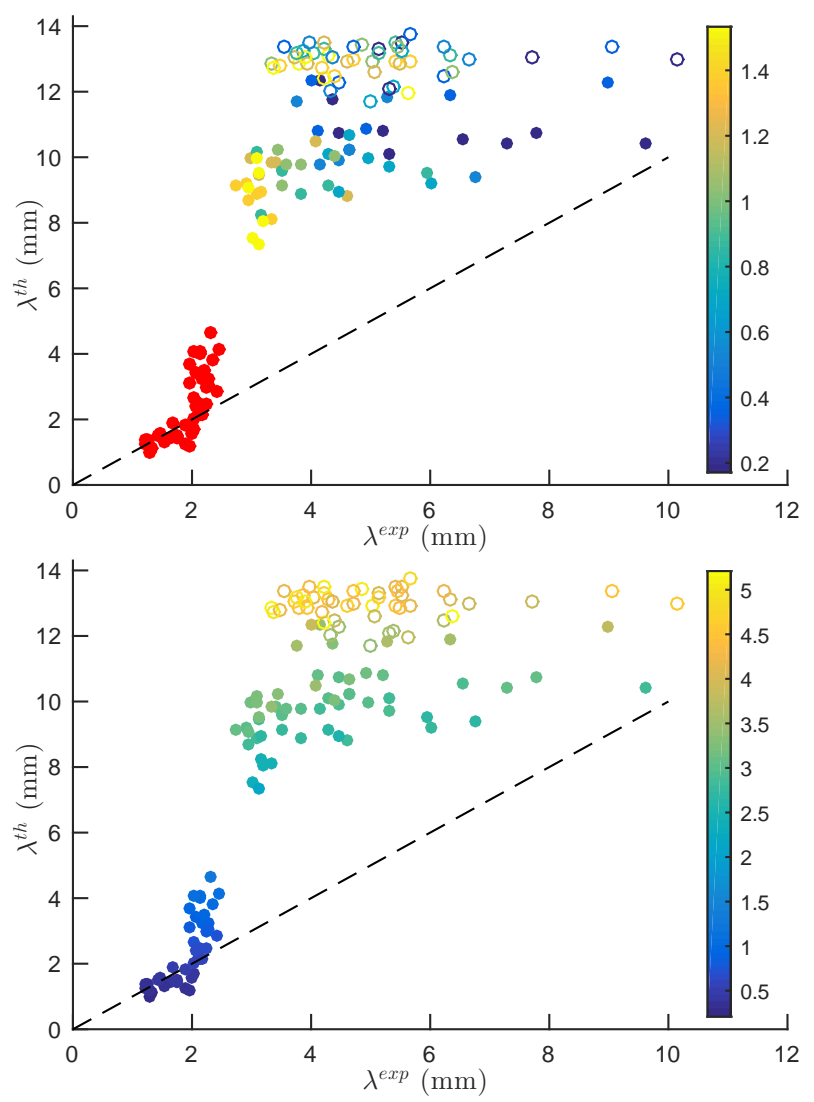

FIG. 13. Theoretical wavelength as a function of the experimental value shown in Fig. $5(\circ), 6(\bullet)$ and $7(\bullet)$ with the same parameters as in Fig. 12. (Top) The color chart shows the effective gravity value in $\mathrm{m} / \mathrm{s}^{2}$, red corresponding to $9.8 \mathrm{~m} / \mathrm{s}^{2}$. (Bottom) The color chart shows the Frankel's film height $d_{2}$ in $\mathrm{mm}$. meniscus and the thick film, may thus be $\partial_{z} u=u / \delta_{m}$. It reduces to the condition $u=0$ at small $\delta_{m}$ and to the alternative limit $\partial_{z} u=0$ at large $\delta_{m}$. This weaker constraint at the upper boundary would reduce the influence of the finite film size on the fastest wavelength and may lead to a better agreement with our observations.

\section{CONCLUSION}

In this paper, we describe quantitatively for the first time the Rayleigh Taylor instability arising in foam film when a thick film is produced on top of a thin film. As this situation is induced by any extension of a nonhorizontal film, it should also happen in a 3D foam when local deformations are induced by coarsening or external shear. This process should potentially influence the film thickness distribution in 3D foams, and thus the gas diffusion from one bubble to the other and so the coarsening rate, or the energy dissipation induced by film shearing, and thus the apparent viscosity. It is also an original example of purely 2D instability, whose nonlinear evolution may be worth a more extensive study.

\section{APPENDIX : NORMAL STRESS CONTINUITY}

The normal stress in each domain is $K=2 \mu_{s} \partial_{z} w_{i}+\partial_{z, i} \sigma \varepsilon+\delta \sigma_{i}$, so

$$
\begin{aligned}
& k^{2} K=2 k^{2} \mu_{s} \partial_{z} w_{i}+\frac{\rho g h_{i} k^{2}}{n} w_{i}+\rho h_{i} n \partial_{z} w_{i}+\mu_{s}\left(k^{2}-\partial_{z z}\right) \partial_{z} w_{i}= \\
& \frac{\rho g h_{i} k^{2}}{n}\left(A_{i}+B_{i}+C_{i}+D_{i}\right)+\left(3 k^{2} \mu_{s}+\rho h_{i} n\right)\left(k A_{i}-k B_{i}+q_{i} C_{i}-q_{i} D_{i}\right)-\mu_{s}\left(k^{3} A_{i}-k^{3} B_{i}+q_{i}^{3} C_{i}-q_{1}^{3} D_{i}\right)
\end{aligned}
$$

The condition (28) is thus, using $\mu_{s}\left(q_{i}^{2}-k^{2}\right)=\rho h n$ in each phase,

$$
\begin{aligned}
& 0=A_{1}\left(\frac{\rho g h_{1} k^{2}}{n}+2 k^{3} \mu_{s}+\rho h_{1} n k\right)+B_{1}\left(\frac{\rho g h_{1} k^{2}}{n}-2 k^{3} \mu_{s, 1}-\rho_{1} h_{1} n k\right)+ \\
& C_{1}\left(\frac{\rho g h_{1} k^{2}}{n}+2 k^{2} q_{1} \mu_{s}\right)+D_{1}\left(\frac{\rho g h_{1} k^{2}}{n}-2 k^{2} q_{1} \mu_{s}\right)+ \\
& -A_{2}\left(\frac{\rho g h_{2} k^{2}}{n}+2 k^{3} \mu_{s}+\rho h_{2} n k\right)-B_{2}\left(\frac{\rho g h_{2} k^{2}}{n}-2 k^{3} \mu_{s}-\rho h_{2} n k\right) \\
& -C_{2}\left(\frac{\rho g h_{2} k^{2}}{n}+2 k^{2} q_{2} \mu_{s}\right)-D_{2}\left(\frac{\rho g h_{2} k^{2}}{n}-2 k^{2} q_{2} \mu_{s}\right)
\end{aligned}
$$


Making finally the transformation (38) - $\rho g k^{2} /(2 n)\left(h_{1}+h_{2}\right)(25)-2 k^{2} \mu_{s}(26)$ we get eq. (30).

\section{APPENDIX 2}

The determinant of the system (33) can be reduced by the transformation $C_{2} \rightarrow C_{2}-C_{1}, C_{3} \rightarrow C_{3}+C_{1}$ and $C_{4} \rightarrow C_{4}-C_{3}$, leading to

$$
\begin{array}{|cccc}
1 & 0 & 0 & 0 \\
k & q_{1}-k & 2 k & q_{2}-k \\
2 k^{2} \mu_{s} & \mu_{s}\left(q_{1}^{2}-k^{2}\right) & 0 & -\mu_{s}\left(q_{2}^{2}-k^{2}\right) \\
X & \alpha_{1} & R-\left(\alpha_{1}+\alpha_{2}\right) & \alpha_{2}
\end{array} \mid=
$$

[1] S. Cohen-Addad, R. Höhler, and O. Pitois, Annu. Rev. Fluid. Mech. 45, 241 (2013).

[2] N. D. Denkov, S. Tcholakova, K. Golemanov, K. P. Ananthapadmanabhan, and A. Lips, Phys. Rev. Lett. 100, 138301 (2008).

[3] J. M. Chomaz and B. Cathalau, Phys. Rev. A 41, 2243 (1990).

[4] H. Kellay, Phys. Fluids 29, 111113 (2017).

[5] V. Bergeron, J. Phys.: Condens. Matter 11, R215 (1999).

[6] C. Stubenrauch and R. V. Klitzing, J. Phys.: Condens. Matter 15, R1197 (2003).

[7] K. J. Mysels, K. Shinoda, and S. Frankel, Soap films: Study of their thinning and a bibliography (Pergamon, New-York, 1959).

[8] H. N. Stein, Adv. Colloid Interface Sci. 34, 175 (1991).

[9] V. Carrier, S. Destouesse, and A. Colin, Phys. Rev. E 65, 061404 (2002).

[10] N. Adami and H. Caps, EPL 106, 46001 (2014).

[11] J. Seiwert, R. Kervil, S. Nou, and I. Cantat, Phys. Rev. Lett. 118, 048001 (2017).

[12] A. Aradian, E. Raphaël, and P.-G. de Gennes, Europhys. Lett. 55, 834 (2001).

[13] S. A. Koehler, S. Hilgenfeldt, E. R. Weeks, and H. A. Stone, Journal of colloid and Interface Science 276, 439
The condition (34) is finally obtained by a development of the determinant along the last line.

\section{ACKNOWLEDGMENTS}

This project has received funding from the European Research Council (ERC) under the European Union's Horizon 2020 research and innovation programme (grant agreement No 725094). We thanks H. A. Stone and H. Kellay for enlightening discussions.
(2004).

[14] O. Pitois, C. Fritz, and M. Vignes-Adler, J. Colloid Interface Sci. 282, 458 (2005).

[15] O. Pitois, N. Louvet, and F. Rouyer, Eur. Phys. J. E 30, 27 (2009).

[16] A.-L. Biance, A. Delbos, and O. Pitois, Phys. Rev. Lett. 106, 068301 (2011).

[17] J. Seiwert, M. Monloubou, B. Dollet, and I. Cantat, Phys. Rev. Lett. 111, 094501 (2013).

[18] C. Derec et al., EPL 112, 34004 (2015).

[19] A. Cohen, N. Fraysse, and C. Raufaste, Phys. Rev. E 91, 053008 (2015).

[20] J. Seiwert, J. Pierre, and B. Dollet, J. Fluid Mech. 788, 183 (2016).

[21] A. Bussonnière, E. Shabalina, X. Ah-Thon, M. L. Fur, and I. Cantat, arXiv preprint arXiv:1902.07076 (2019).

[22] G. I. Taylor, Proceedings of the Royal Society of London. Series A. Mathematical and Physical Sciences 201, 192 (1950).

[23] W. Drenckhan et al., Phys. Fluids 19, 102101 (2007).

[24] M. A. Rutgers, X. I. Wu, R. Bhagavatula, A. A. Petersen, and W. I. Goldburg, Phys. Fluids 8, 2847 (1996).

[25] S. Chandrasekhar, Hydrodynamic and hydromagnetic stability (Courier Corporation, 2013). 